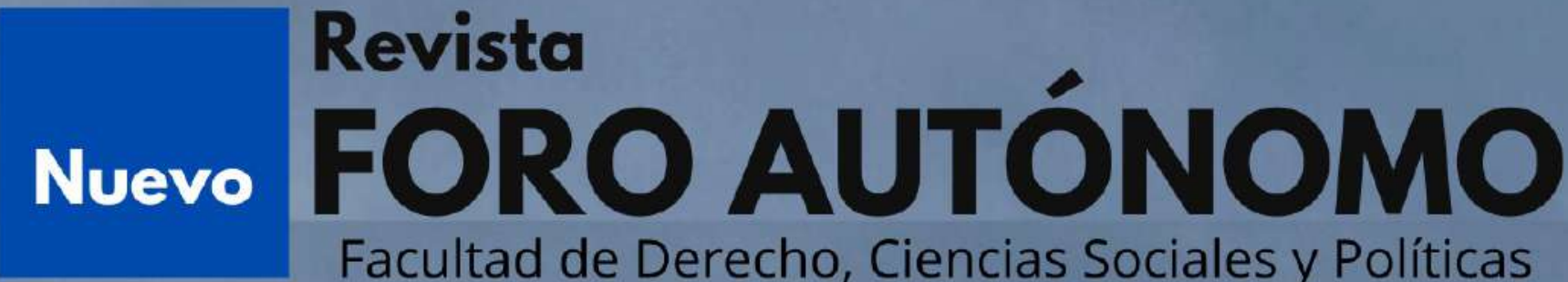

Popayán-Colombia Vol 2, Núm 2 • julio - diciembre de $2021 \quad$ ISSN: 2711- 4856 (En línea) Corporación Universitaria Autónoma del Cauca https://nfa.uniautonoma.edu.co

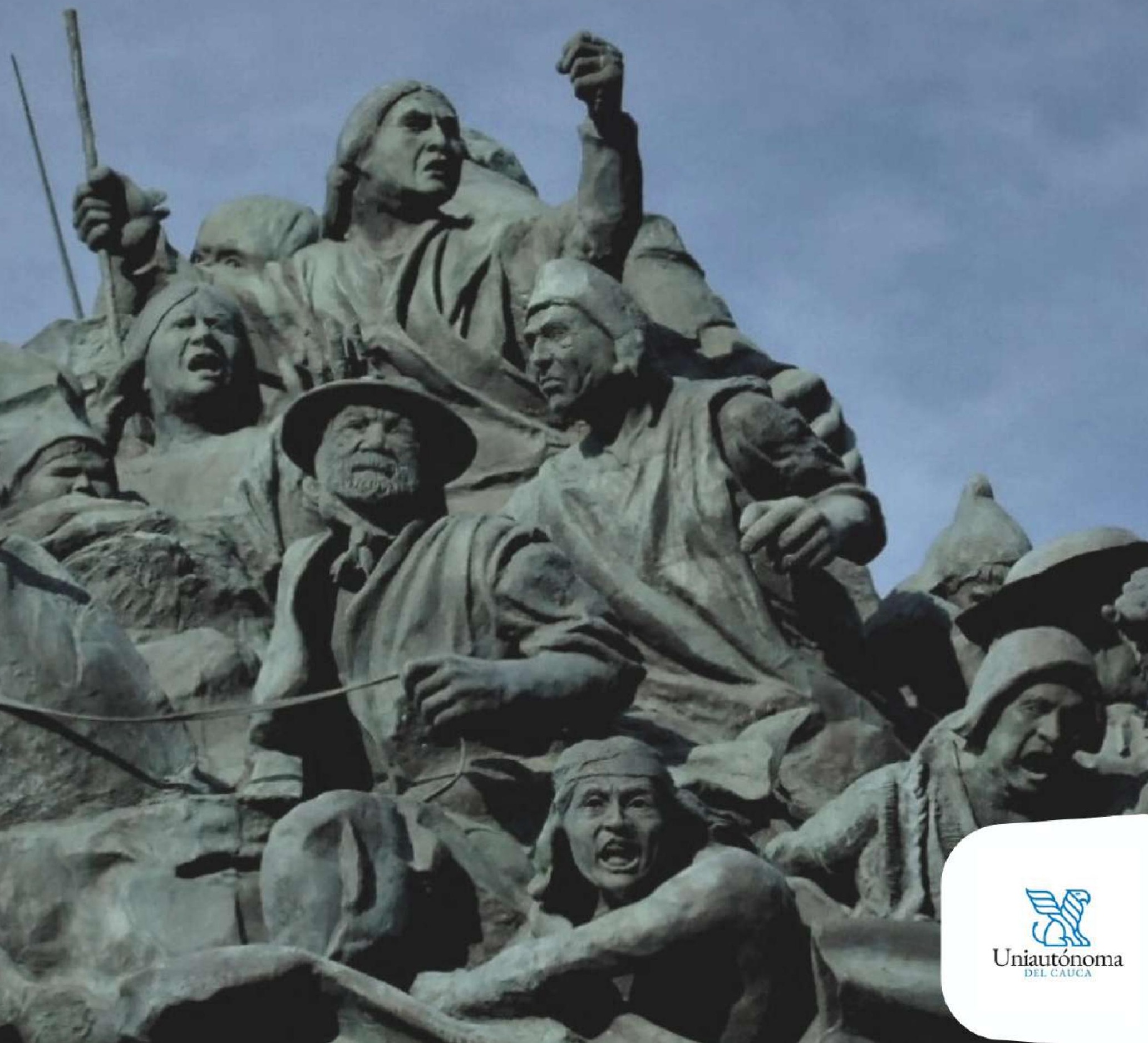





\section{Nuevo FORO RUTÚ, AUOMO}

Volumen 2 - Número 2 • julio-diciembre de 2021 • ISSN: 2711-4856 (en línea)

Corporación Universitaria Autónoma del Cauca

Facultad de Derecho, Ciencias Sociales y Políticas

Sello Editorial Uniautónoma del Cauca

Popayán (Cauca - Colombia)

Correo electrónico: revistaforoautonomo@uniautonoma.edu.co

Website: https://nfa.uniautonoma.edu.co

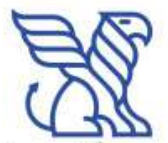

Uniautónoma

Diagramación: Sello Editorial Uniautónoma del Cauca

Foto de portada: Juliana Rodríguez Arango

\section{Comité Científico}

Dra. Lyda Teresa Córdoba Hoyos

Universidad del Valle

Dr. Luis Eduardo Ruano Ibarra

Universidad Cooperativa de Colombia

Dr. Ramsés López Santamaría

Corporación Universitaria Autónoma del Cauca

Dr. Gildardo Vanegas Muñoz

Universidad del Cauca

\section{Comité Editorial Central}

Dr. Daniel Augusto Mantilla Sandoval

Rector Corporación Universitaria Autónoma del Cauca

Dr. Juan Pablo Sterling Casas

Director Revista Nuevo Foro Autónomo

Decano Facultad de Derecho, Ciencias Sociales y Políticas

Mg. William Darío Chará Ordóñez

Editor Revista Nuevo Foro Autónomo 


\section{Contenido}

Las condiciones de posibilidad de Justicia, un análisis

desde la desigualdad social y económica

Julián David Guachetá Torres

La justicia transicional en la Jurisdicción Especial para la Paz

Laura Valentina Barrero Jiménez

Aproximación a los hábitos y técnicas de estudio en instituciones educativas públicas, un estudio de caso en Popayán".

Paola Andrea Gómez Quirós

Empresas transnacionales comosujetos de derecho internacional

35

Victoria Eugenia Paz Trullo

Lucy Esmeralda Paz Trullo

Excepciones y limitaciones del uso de la

fuerza: autonomía universitaria y derecho a la protesta

53

Rubén Darío Chaves Canabal 


\title{
Las condiciones de posibilidad de Justicia, un análisis desde la desigualdad social y económica
}

\section{The conditions of possibility of Justice. Analysis of social and economic inequality}

\author{
Julián David Guachetá Torres* \\ Corporación Universitaria Autónoma del Cauca \\ correo electrónico: julian.guacheta.t@uniautonoma.edu.co
}

Recibido: 28/10/2021 • Revisado: 06/11/2021 • Aceptado: 10/12/2021

DOI: https://doi.org/10.46571/NFA.2021.2.2.1

Cómo citar: Guachetá, J. (2021). Las condiciones de posibilidad de Justicia, un análisis desde la desigualdad social y económica. Revista Nuevo Foro Autónomo, 2 (2), pp. 5-15. DOI: https://doi.org/10.46571/NFA.2021.2.2.1

\section{Resumen}

Este trabajo tiene como objetivo analizar las condiciones de posibilidad de la justicia, desde la desigualad social y económica. Para lo anterior, se abordaron las ideas de justicia de Jhon Rawls, Robert Nozick, Amartya Sen y Martha Nussbaum, entre otros, de igual forma, se plantea la existencia de niveles de desaprobación ante hechos similares, como una forma de hacer referencia al dolor y rechazo selectivo a nivel social y político respecto a hechos graves, pero, que afectan a personas a quienes su dolor o sufrimiento es permitido. Así mismo, se presentan datos relacionados con los procesos de desigualdad económica en el mundo y en América Latina, los cuales permiten evidenciar que la declaración masiva de derechos, en las constituciones por ejemplo, no es suficiente para el goce efectivo de los mismos, de similar modo, que el modelo económico y social, termina definiendo buena parte de la posibilidad para la existencia de justicia real.

Palabras clave: justicia, desigualdad, injusticia, derecho, modelo económico.

\begin{abstract}
The purpose of this paper is to analyze the conditions of possibility of justice, from the perspective of social and economic inequality. For this purpose, the ideas of justice of Jhon Rawls, Robert Nozick, Amartya Sen and Martha Nussbaum, among others, were approached. Similarly, the existence of levels of disapproval of similar events is proposed as a way of referring to pain and selective rejection at the social and political level with respect to serious events, but which affect people whose pain or suffering is permitted. In addition, data related to the processes of economic inequality in the world and in Latin America are presented, which show that the massive declaration of rights, in constitutions for example, is not sufficient for the effective enjoyment of these rights, in the same way that the economic and social model ends up defining a good part of the possibility for the existence of real justice.
\end{abstract}

keywords: Armed conflict, victims, peace, transitional justice.

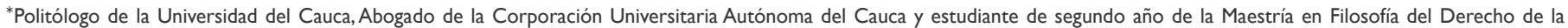

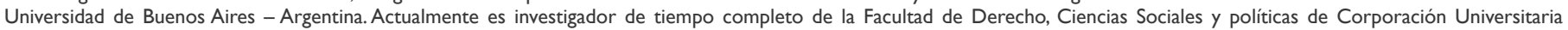
Autónoma del Cauca. ORCID: https://orcid.org/0000-0003-1697-9463 


\section{Introducción}

La justicia puede catalogarse como el tema central en el estudio del derecho, su amplitud es tal, que pese su reconocida relevancia aún no hay acuerdo respecto a su significado y ni la forma de alcanzarla, lo anterior no implica la ausencia de esfuerzos ni aportes de distintos autores, sino que pese a tales, desde la academia no solo relacionada con el derecho, la justicia sigue siendo un tópico rico en discusión, del cual se siguen extrayendo importantes ideas, y abriendo interesantes discusiones frente a lo que realmente implica la justicia en las sociedades actuales, marcadas por avances tecnológicos y mejoras en la calidad de vida sin presentes, pero, al mismo tiempo, marcadas por brechas de desigualdad cada vez más amplias, dentro de un modelo de mercado que propicia e incentiva la desigualdad económica.

Este trabajo tiene como objetivo analizar las condiciones de posibilidad de la justicia, desde la desigualad social y económica, elementos claves e indispensables para hablar realmente de esta, entendida como un estado de las cosas que requiere de cierto equilibrio, el cual se ve afectado en presencia de la desigualdad misma, en este caso a nivel social y económico, esto implica de entrada entender a la justicia desde la visión de Amartya Sen, basada en condiciones materiales reales y no por aquella justicia ideal, que plantea nuevas interpretaciones del contrato social o posiciones originales como lo hace Rawls. De ahí que la justicia no solo es un tema del derecho, ya que para comprender su dimensión real hay que ver más allá de lo netamente jurídico o normativo, pues las condiciones de posibilidad de la justicia se hayan también en la configuración social y económica de los Estados.

Bajo la perspectiva anterior, se tiene que los criterios de lo bueno y lo malo, lo justo y lo injusto, tienen raíces en las configuraciones sociales y culturales de las sociedades, razón por la cual, aquello que es bueno para una sociedad X, (tener más de una esposa) puede ser algo malo para una sociedad Y, este pequeño ejemplo, pretende poner de presente que la universalización de los criterios que definen algo como bueno o justo, son complejos de definir. En este punto, surge un problema clave para entender a la justicia, es decir, realmente existen actos de justicia o lo que hace que un acto sea injusto es la valoración que se hace del mismo, esta confrontación se produce desde el escepticismo moral y el realismo moral, en otras palabras, implica preguntarse por el estatus ontológico de los valores relacionados con la justicia, así como por el estatus epistemológico de dichos valores, y sí realmente se pueden conocerse de manera absoluta, se trata de posturas que buscan comprobar o no la existencia de "verdad" en las valoraciones sobre los actos. En este panorama, es oportuno mencionar lo dicho por Barbarosch, en el sentido de que "Adherir a una concepción de justicia no significa necesariamente defender su verdad moral" (2013), de ahí que, el escepticismo moral no sea un impedimento para indagar por las condiciones de posibilidad de la justicia en los términos propuestos.

Habiendo hecho la salvedad anterior, se debe hacer mención a ciertos fenómenos sociales y culturales que dificultan el análisis objetivo de la justicia, estos tienen que ver con lo que se puede denominar como niveles de desaprobación ante hechos similares, los cuales hacen que haya distintas muestras de rechazo o de sufrimiento según las personas que se vean afectadas por distintos actos humanos o hechos naturales, casi como un dolor selectivo,

Revista Nuevo Foro Autónomo • Volumen 2 - Número 2 julio-diciembre de 2021 • pp. 29-46 • ISSN: 2711-4856 (en línea) 
como muestra de esto, se puede ver que hay muertos que merecen ser llorados y otros que no, en razón de su nacionalidad, color de piel, orientación sexual o religiosa etc., pensemos en los migrantes africanos o latinoamericanos que día a día mueren intentando llegar a países en donde esperan mejorar sus condiciones de vida, se tiene entonces que "Al menos 1.146 personas perdieron la vida intentando llegar a Europa por vía marítima en los primeros seis meses de 2021" (OIM, 2021), pese a estos números realmente trágicos, se puede ver casi un silencio cómplice de los países directamente implicados.

La normalización del sufrimiento de cierto tipo de personas, hace ver a la justicia desde un plano casi que metafísico. Pensemos en otra situación que sí conmocionó al mundo, y tiene que ver con el atentado de 2015 contra Charlie Hebdo, el satírico semanario francés, evento que dejo 12 víctimas fatales a manos de extremistas que hacían parte de Al-Qaeda en la Península arábiga, este hecho deleznable contra ciudadanos franceses ocasionó que unas 2'000.000 de personas, dentro de las cuales se incluyen más de 40 líderes mundiales se unieran a una marcha de unidad nacional, en total, cerca de 3'700.000 personas participaron de las manifestaciones celebradas en toda Francia, de igual forma, se presentaron amplias muestras de apoyo en redes sociales en donde las personas aplicaban una marca de agua de la bandera francesa a su foto de perfil en Facebook, indicando su repudio a los atacantes o el apoyo a las víctimas, lo anterior sin mencionar la amplia cobertura mediática mundial (Gómez, et al, 2016), que recriminaba el hecho de que ciudadanos francés debieran de soportar este tipo de actos. Se tiene entonces, que la comunidad internacional, no tiene criterios similares para juzgar el sufrimiento humano, es válido o casi que permitido que 1.146 seres humanos mueran ahogados intentando escapar de sus países, pero inadmisible que 12 ciudadanos franceses pierdan sus vidas a costa de radicales musulmanes.

Lo anterior, se puede ver representado a partir de la exclusión del otro, la otredad o lo exótico puede violentarse. Los procesos de exclusión de cuerpos han sido normalizados al largo de la historia y en distintos sistemas jurídicos, el caso de los judíos en Venecia a quienes desde el final del siglo XIV hasta comienzos del XVI no se les permitía residenciarse fuera del gueto de Venecia y en donde la ley reglaba su relacionamiento con la población cristiana, indicando, por ejemplo, la prohibición de estrechar la mano o mantener un contacto directo con un judío, lo anterior es sólo un ejemplo que metodológicamente permite hacer los análisis respecto a este fenómeno social, estas exclusiones pueden tener diferentes sustentos, por ejemplo de tipo religioso, ético o cultural, lo cierto, es que el hecho de rechazar un cuerpo no es un fenómeno espontaneo, sino construido socialmente. Precisamente, estas construcciones sociales son las que buscan ser descifradas, y para lo cual, se deben distinguir las construcciones de primer grado, es decir, los actos de la cotidianidad y las construcciones de segundo grado, que hacen referencia a una visión "científica" que se nutre de las primeras y permiten interpretar a la realidad (Schuster, 2001), estos procesos de interpretación deberán tener como eje al lenguaje, visto como una construcción social que permite comprender los procesos sociales.

De esta forma, es claro que la exclusión de los cuerpos es un proceso de normalización de la exclusión, y como afirma Alicia Ruiz, el prejuicio religioso no es un ejercicio de racionalidad (Ruiz, 2014), esto se puede extrapolar a la exclusión sistemática e institucional del pobre y el marginado, en estas dinámicas de exclusión, la justicia como aspiración sufre 
una reconfiguración, por lo que salta en escena un concepto importante y es el de intersubjetividad, como superación de la objetividad, ya que hablar en términos de objetividad implica desconocer las relaciones sociales que atraviesan a los sujetos y configuran las interacciones humanas, la intersubjetividad permite tener conciencia de que los acuerdos parciales sobre determinados temas son los que construyen eso que llamamos realidad y en este caso justicia, la cuestión aquí, es el tipo de acuerdo global y estatal de lo que implica la justicia.

Los ejemplos de los migrantes y el atentado contra Charlie Hebdo no deben entenderse como un reproche hacia quienes rechazan la violencia radical y extremista, o quienes aplicaron la marca de agua a su foto de perfil en Facebook, ya que como se mencionó, se trata de hechos completamente repudiables, lo que se busca poner de presente, es que dicho dolor y rechazo no son aplicables a otro tipo de situaciones que pueden llegar a ser peores en términos de garantías humanas vulneradas. De ahí que, se pueda afirmar, que la realidad está compuesta por hechos y tales hechos son valorados como buenos y malos, lo cual se acercaría a una visión escéptica de la ética, sin ser esta la intención, es así, como a partir de ciertas construcciones sociales y culturales, se establecen parámetros que definen si una acción violenta contra un ser humano es aceptada o permitida o puede ser valorada como buena, mala, justa o injusta.

Luego de explicar lo que se mencionó como niveles de desaprobación ante hechos similares, es menester poner de presente sus implicaciones para la justicia, se tiene entonces, que la justicia como aspiración de toda institución en términos de Rawls, termina siendo afectada por lo que es permitido o rechazado en una sociedad, el ejemplo del atentado a Charlie Hebdo y los cientos de migrantes africanos muertos, se puede encontrar al interior de los países latinoamericanos, en donde las vicisitudes de las grandes capitales ocupan un lugar de relevancia superior al que ocupan los problemas de los pueblos y comunidades del interior de los países, sumidas generalmente en condiciones de pobreza, falta de servicios básicos, garantías humanas etc., sin que esto implique, que en las grandes ciudades y en las periferias de estas principalmente, también se produzcan este tipo de dificultades, el hecho de que el sufrimiento de unos ciudadanos sea más o menos valorado que el de otros es un obstáculo de la justicia.

Las ideas hasta aquí planteadas se pueden entender desde varias orillas teóricas y metodológicas, una de ellas es la ideológica y las relaciones de dominación que explicarían los procesos de exclusión y demás, en ese caso se afirma que, ante un ejercicio hegemónico, surge un proceso contra hegemónico, y ambos están atravesados por la ideología, es decir, ideas que expresan y promueven los intereses principales de las partes en conflicto, se trata entonces del sustrato que fundamenta su acción. En este punto, surge el concepto de consenso, el cual es fundamental para la ideología en Gramsci, pues éste crea y masifica determinada ideología sustentando además su existencia, ese consenso es, por tanto, más efectivo que la fuerza o coacción, ya que, si existe consenso sobre determinada ideología, su defensa estará en cabeza de los oprimidos (Gramsci, 1978), en este caso, se normalizan ciertos actos, por ejemplo, de desigualdad económica desbordada y la vulneración de derechos, que luego afectan a la justicia como aspiración, entendida como una mera idea, pero no como una práctica plausible. 
Por esta razón, un punto de vista político puede ser bastante exacto o verdadero en su contenido, por ejemplo, frente a los ideales justicia y, sin embargo, completamente ideológico, o de modo contrario, la idea que un punto de vista político en su contenido social puede estar completamente equivocada sin que haya nada "ideológico" en él (Žižek, 2003, p. 13).

\section{Resultados}

Lo dicho hasta aquí, conduce a dos caminos distintos, pero que al mismo tiempo son claves para el estudio de la justicia, por un lado, una visión abstracta, que ha sido catalogada como ideal y una visión real en términos de aplicación práctica en la realidad material, la primera de estas, se toma desde Rawls, aunque con ciertos matices, por ejemplo, que su teoría de la justicia no solo se agota en lo ideal, sin embargo, su relectura del contrato social y de la posición original como explicación o método para encontrar los valores fundamentales que debe perseguir una sociedad, hacen que sus ideas sirvan de fundamento teórico de la justicia, y más si se considera que "El sentido de justicia es definido por Rawls como la capacidad moral que tenemos para juzgar cosas como justas, apoyar esos juicios en razones, actuar de acuerdo con ellos y desear que otros actúen de igual modo" (Francisco, 2006, p. 4).

El otro camino, que se debe mencionar es el de la justicia vista como acción, en este caso Amartya Sen entra en un dialogo con Rawls, de lo anterior, surgen críticas a su visión, puesto que el autor indio defiende la idea de una la justicia basada en realizaciones, dejando de lado la justicia ideal o trascendental basada en las instituciones de Rawls, pese a que reconoce el rol fundamental del Estado. Para su teoría es clave, el concepto de injusticia como el que da pie precisamente, a la búsqueda de la justicia, de ahí que, el trabajo de Sen se enfoque en los acuerdos razonados para reducir la injusticia, más que en idealizar, indicando que los acuerdos pueden ser alcanzados, más allá de las diferencias posibles entre los interlocutores de una sociedad, se requiere entonces de un ideal, pero lo crucial no es el ideal, sino la reducción de las injusticias de ahí que su idea de la misma se afinque en la realidad de los individuos y en la razón (Sen, 2011).

Uno de los puntos que unen al pensamiento de Sen y Rawls tiene que ver con el papel central de la razón en la comprensión de la justicia, en este caso, la razón como medio para alcanzarla, por esto, se afirma que la esperanza en un mundo menos injusto reside en la discusión pública razonada, esta afirmación de plano se aleja de la creencia de que las cuestiones sobre valores tengan solo una respuesta verdadera, la discusión precisamente radica en la mediación de estas respuestas respecto a los valores (Colmenarejo, 2013), dicha idea de Sen remite al velo de la ignorancia, o posición original descrito por Rawls, el cual plantea un estado imaginario de ignorancia, puntualmente en lo que refiere a los intereses personales y las posturas de lo que significa una buena vida, esta posición original, es un ejercicio abstracto permitiría tomar un conjunto de principios de justicia por unanimidad (Rawls, 1971). Las ideas de justicia tanto de Rawls como de Sen, no son excluyentes entre sí, por el contrario, Sen expresó siempre admiración por el trabajo del teórico de Harvard y ambos son conscientes de la responsabilidad del Estado en lo que respecta a lograr un mínimo de justicia social en los eventos en que el sistema económico no lo permita. 
Este trabajo precisamente hace hincapié en las profundas limitaciones de la justicia ante escenarios de desigualdad social y económica desbordada, en otras palabras, que las condiciones de posibilidad de la justicia radican en la garantía de necesidades básicas, ya que, de lo contrario, la justicia como ideal será un discurso desprovisto de una aplicación real que tenga impacto directo en todos los ciudadanos, si se analizan datos básicos a nivel económico en el mundo, se verá que "Desde 2015, la población más rica, que supone un $1 \%$, posee más riqueza que el resto del planeta" (Oxfam, 2021), y en 2019, "los 2.153 milmillonarios que hay en el mundo poseían más riqueza que 4.600 millones de personas" (2021), estos datos son muestra de que la desigualdad es un fenómeno cada vez más grave y contra el cual es poco lo que se puede hacer, desde quienes no tienen capacidad de decisión.

Si se analiza la situación en América Latina y el Caribe, se tiene que "el $20 \%$ de la población concentra el 83 \% de la riqueza" (Oxfam, 2021), con respecto al escenario opuesto, se puede apreciar un aumento notable de la pobreza extrema, pues "En 2019, 66 millones de personas, es decir, un 10,7 \% de la población vivía en extrema pobreza, de acuerdo a datos de la Comisión Económica para América Latina y el Caribe" (Oxfam, 2021), estos datos que se presentan no son algo nuevo, de hecho año a año, los niveles de desigualdad se acrecientan en el mundo y en la región. Lo relevante para este trabajo, es señalar que la justicia como ideal del derecho requiere de condiciones de igualdad no solo normativa en términos de reconocimiento de derechos, sino de igualdad o por lo menos de garantías materiales básicas sin las cuales, la justicia para quienes no tienen estas garantías será un discurso vacío al cual no se puede acceder.

Al escenario anterior, debe sumarse la creciente injusticia social en las sociedades latinoamericanas, acrecentada por la pandemia y la desaceleración económica, esto no hace sino poner en evidenciar la imposibilidad de la justicia como práctica bajo tales condiciones, por el contrario, la injusticia social o la carencia de condiciones materiales mínimas, termina romantizando a la pobreza, lo cual crea un terreno fértil para fenómenos como el coaching y la superación personal, en donde el problema de las condiciones materiales recae en el sujeto que no es capaz de seguir las dinámicas del mercado y "superarse" pese a que no tiene condiciones mínimas para desarrollar su vida con dignidad, de esta forma, detrás de discursos individualistas y no sociales o colectivos, se camuflan las incapacidades estatales y del sistema económico de proporcionar condiciones de igualdad o en último término de equidad, dejando un escenario que Chul Han describe de manera muy clara, en donde el sujeto se explota así mismo creyendo que se está superando, tomando en cuenta las oportunidades que el sistema económico le ofrece (2017).

Hablar de justicia no solo implica hablar de derecho o la forma en cómo se resuelven controversias judiciales, el asunto central, se haya en la forma en como la sociedad funciona de manera cotidiana, por ejemplo, con la idea de progreso que está latente en las realidades, la tecnología, los avances en medicina y otros, muestran un contraste entre un futuro prometedor y uno decadente respecto del ser humano, visto como medio y no como fin, pero lo cierto, es que la idea de progreso y evolución en cierto punto llega a ser ilusoria, por lo menos para la gran mayoría de las personas, que como se vio, se enfrentan a la ausencia material de condiciones y por ende de verdadera justicia. 
Sumado a lo anterior, el problema de la justicia en términos judiciales, radica en la estructura y funcionamiento de los ordenamientos jurídicos latinoamericanos, resulta contradictorio que nunca como actualmente se tenga tal cantidad de derechos declarados, garantías reconocidas y un volumen inmenso de derecho positivizado en disposiciones normativas nacionales e internacionales, sin embargo, esto no ha sido óbice para que nunca como actualmente existan brechas entre unos seres humanos sobre otros. Si se toma el ejemplo de la acumulación de riqueza como ya se evidenció, se aprecia que la declaración de la igualdad en las constituciones modernas no garantiza realmente que exista igualdad, sin embargo, el derecho parece agotarse en la declaración masiva de derechos, pareciera que al tiempo en que se reconocen garantías, las desigualdades se acrecentaran, quizá uno de los responsables puede ser el constitucionalismo moderno que declara derechos de manera masiva pero no tiene la capacidad para lograr su disfrute.

Todo lo anterior, se desarrolla cuando se atraviesa un periodo de avance tecnológico, que, de nuevo, crea la falsa ilusión de progreso por lo menos en América Latina, "se estima que en 2020 la tasa de pobreza extrema se situó en 12,5 \% y la tasa de pobreza alcanzó el 33,7 \% de la población" (CEPAL, 2021). Se tiene que "el total de personas pobres ascendió a 209 millones a finales de 2020, 22 millones de personas más que el año anterior. De ese total, 78 millones de personas se encontraron en situación de pobreza extrema, 8 millones más que en 2019" (2021). Se está ante una situación realmente compleja, que tiene efectos en la forma en como del derecho funciona y por ende la justicia. La injusticia en términos de Sen, es la que incentiva la búsqueda de la justicia, y en las prácticas cotidianas es donde se haya el derecho aplicado, que crea procesos de impunidad. Vincular estos problemas es fundamental para entender el asunto de manera holística, las explicaciones deben partir de la realidad, más allá de teorías sofisticadas que explican la forma como los sistemas jurídicos se desarrollan y de la lógica que simboliza y representa los posibles escenarios de aplicación de la norma.

Si el derecho deja de tener en cuenta las condiciones de posibilidad de la justicia no es posible analizarla en sentido amplio, las reglas de interpretación, la valoración de las pruebas, la construcción o redacción de las normas, y los procesos judiciales en sí, están profundamente influenciados por las dinámicas sociales, económicas y culturales de quienes intervienen en el proceso, tomemos como ejemplo a un congresista o diputado que afronta un proceso judicial por peculado, y que tiene a su disposición un gran capital económico para contratar a los mejores servicios jurídicos, ante este escenario, tiene mejores posibilidades de salir impune del proceso, o por lo menos con una condena favorable. Si se hace este ejercicio con un ciudadano pobre que hurto las pertenencias a un par de personas en un callejón, y que a quien se le asigna un defensor de oficio, el escenario será distinto, pese a que el peculado seguramente habrá hecho más daño en la sociedad. Lo que se quiere dejar en claro con este ejemplo, no es una defensa del carterista, sino evidenciar que ante dos hechos que ameritan sanción, y que causan afectación a la sociedad las posibilidades de condena son sumamente diferentes, se debe hacer mención, además, que el deficiente funcionamiento de la estructura judicial, la masiva acumulación de procesos, la corrupción y otros factores, crean escenarios de impunidad que incrementan la sensación de injusticia en la comunidad. 
En este punto, es válido traer a colación a Rawls, y un elemento de su teoría de la justicia, puntualmente en lo que respecta a la desigualdad, en donde indica, que esta es admisible siempre que beneficie a los más necesitados (Rawls, 1971), por lo que, en esencia, la justicia no es un derecho en sí, sino un ejercicio político que requiere del derecho para lograr su materialización, esta idea es clave para interpretar la justicia de Rawls, quien logra dar el salto de la consideración iusnaturalista de la justicia ya que "no se presenta ya con una pretensión ontológica de origen divino o racional superior al fenómeno jurídico positivo" (Barbarosch, 2013), el hecho de entender a la justicia como algo más que derecho implicó que la política y la economía tuvieran mucho que decir de la misma, de ahí que el derecho pueda ser derecho sin que necesariamente sea justo, la labor están en transformar al derecho, la justicia como criterio de validez puede ser una opción, pero en este caso, la complejidad esta en definir a la justicia y lo justo e injusto para el derecho.

Ante este escenario, se debe indicar que autores como Nozick apoyado en Locke, defienden la idea de la propiedad como un derecho natural, el cual es fundamental para la organización social, en ese sentido, los hombres son dueños de sí mismos, por lo que la sociedad no puede utilizarlos como medios, tomando esta visión de dignidad kantiana, se argumenta que si un hombre tiene talento y gana más dinero, no se lo puede someter a pagar más impuestos para mejorar la vida de los demás que no tienen ese talento, ya que se vulnera su derecho natural (Nozick, 1988), estas ideas que se instalan en el liberalismo clásico han sido el sustento no solo del modelo económico actual, sino de la visión de justicia, en donde las brechas de desigualdad son permitidas, siempre que se desarrollen en el marco de la libre competencia y el libre mercado, es importante indicar que el pensamiento de Nozick en su libro Anarquía Estado y Utopía de 1974, es una réplica directa a la teoría de la justicia de Rawls de 1971, en donde se busca dentro de lo que se puede denominar como liberalismo igualitario, un modelo de justicia basado en los principios de libertad, de diferencia e igualdad de oportunidades, los cuales van a ser interpretados de distinta manera por Nozick.

Uno de los asuntos claves para justificar o no a las diferencias dentro de una sociedad, en términos económicos y sociales, por ejemplo, tiene que ver con la redistribución de la riqueza de unos, ante la pobreza o carencia de otros, en otras palabras, si la riqueza de un grupo reducido debe soportar por medio de mayores impuestos, que otro grupo de ciudadanos mejoren sus condiciones. Ante este panorama, se tienen dos posturas claras, por un lado, a la visión de Rawls y por otro, la visión de Nozick, quienes difieren diametralmente sobre las diferencias permitidas y la redistribución de la riqueza.

A partir de lo anterior, se tiene a una de las primeras diferencias entre dichos autores, tiene que ver con los talentos dentro de una sociedad, talentos que permiten a una persona, ganar mucho más dinero que otras, o ser más exitosa que otras, talentos que se desarrollan de manera natural o producto del esfuerzo personal, en la visión de Rawls, dichos talentos hacen parte del acervo común de la sociedad, por lo que estos deben ser puestos al servicio de los menos talentosos, ya que como se vio, la diferencia para Rawls es admitida siempre que beneficie a los menos favorecidos en una sociedad, de ahí que, la redistribución de la riqueza es una necesidad para que exista libertad e igualdad de oportunidades y por ende pueda existir justicia (Rawls, 1971). 
En el pensamiento de Nozick, los talentos no hacen parte del acervo común, de hecho, pensar en los talentos de esa manera implica una vulneración al derecho natural de la propiedad, derecho intrínseco y que parte de la propiedad sobre el propio ser, su cuerpo y sus capacidades, esto se relaciona con el principio de auto propiedad, Nozick indica que la cooperación es importante dentro de una sociedad, pero al ser esta una carga impositiva implica la vulneración de garantías superiores, y se estaría hablando de otra forma de esclavitud moderna (1988).

Se tiene entonces, dos formas de entender a las desigualdades y dos formas de enfrentar dicho problema, lo cierto, es que si contrastamos tales ideas con la realidad, se verá que la idea de Nozick ha prevalecido en el modelo actual, las diferencias de salarios entre un futbolista de elite y un profesor de primaria son abismales, pero estructuralmente permitidas, lo que se debe analizar es hasta qué punto las diferencias deben ser avaladas jurídicamente, o si existe un límite para las mismas, por ejemplo, en los eventos en donde los talentos de una persona les permite a sus herederos sin dicho talento, mantener las desigualdades por generaciones, esto lleva a otra discusión y tiene que ver con el rol del Estado en la eliminación o aval de las desigualdades, en donde se puede optar por una visión de Estado mínimo que se encargue de la seguridad, de la justicia en términos judiciales, y otros elementos de funcionamientos, o una visión amplia del Estado, por ejemplo, la que promulga el comunitarismo, en donde este participa activamente influenciando ciertos planes de vida, y se compromete con ideales de bien, como la justicia y la igualdad.

En cuanto a la justicia como aspiración institucional, se debe mencionar que "las divisiones sociales no reflejan las divisiones naturales" (Piscitelli, 1993) o que "El orden social no forma parte de la naturaleza de las cosas" (Berger \& Luckmann, 1993) esto sin duda debe replantear el concepto de normalidad, como un proceso de construcción social de la realidad, esto hace, que a partir de la tipificación reciproca de acciones habitualizadas por diferentes tipos de actores, en donde un sujeto X debe realizar una acción X (1993), se creen estructuras dentro de las cuales se desarrollan las interacciones, estructuras como el matrimonio, las religiones, el Estado etc., influyen en lo que se considera correcto alcanzar en términos de justicia.

Desde otra perspectiva, Cossio plantea la necesidad de entender al derecho más allá de la norma jurídica, lo cual se puede extender a la justicia como algo más que norma jurídica, y si bien aceptaba al positivismo, no creía en la aplicación mecanicista del derecho, ya que este hace parte del relacionamiento social, y por ser de esta forma, está influenciado por los diversos factores que moldean a la conducta humana, así las cosas, la ideología no es la excepción, y para Cossio la ideología es un elemento que influye en el derecho, por lo que debe ser tenido en cuenta en el estudio del mismo (Gassner \& Olechowski, 2014).

Así mimo, Marí expresa a partir de la influencia clara de Foucault, que es necesario incluir en la discusión jurídica al poder, pero no solo entendido como atributo de un sistema jurídico, sino como un elemento de la interacción humana, dicho poder que se encuentra en constante disputa con otros poderes desde la cotidianidad, esto de entrada permite relacionar a lo dicho por Rawls en su teoría de la justicia, en el sentido de llegar a acuerdos desde las diferencias, casi como un manual de negociación, pero siguiendo a Marí, es evidente que en la práctica, estos acuerdos por llamarlos de algún modo, implican procesos 
de dominación o superposición (Marí, 1986), así también, lo destaca Cárcova al señalar una de las críticas a Rawls, en el sentido de querer transformar el reino de la política en un espacio idealizado, en donde los sujetos son despojados de pasiones y creencias molestas, y en donde sus actos son siempre determinados por la razón (2002).

De esta forma, Marí brinda una explicación crucial para el entendimiento de las dinámicas del poder en la sociedad, esto a través de los elementos que crean los dispositivos de poder, es decir, el discurso del orden y el imaginario social, estos elementos, dan cuenta del complejo entramado del que se deberían desprender los llamados acuerdos racionales en Rawls, es decir, no es un proceso de negociación ideal, sino que se trata de profundas interacciones en donde los contextos y las formas de institucionalizar el comportamiento humano son determinantes para superponer o no un determinado parámetro de justicia. De una manera similar, lo entendió Mouffe, es decir, el hecho de concebir lo político como un proceso racional de negociación, implica ignorar la cuestión del poder y el antagonismo que hace parte de lo político por excelencia (Marí, 1986).

La discusión para Marí, no tendría que ver con la teoría de justicia en sí, sino con la forma en como Rawls plantea la posibilidad de acuerdo entre lo diferente, ya que para Marí el imaginario social termina por determinar conductas, por lo que el problema está profundamente relacionado con el hecho de que la negociación implica necesariamente que se desarrollen procesos de dominación por medio de los dispositivos del poder, que son reproducidos de manera inconsciente en la sociedad, por lo que, si no se tiene en cuenta este elemento, es posible que dichos acuerdos racionales y libres no sean posibles.

\section{Conclusión}

Finalmente, y con respecto a las condiciones de posibilidad de la justicia, es menester hacer énfasis en las condiciones materiales de los ciudadanos, ya que, sin estas, las implicaciones reales de la justicia van hacer muy limitadas, las brechas de desigualdad son barreras reales para conseguir sociedades más justas, es oportuno traer la visión de Martha Nussbaum, cuando indica que las teorías de la justicia deben ser abstractas, al mismo tiempo tener un nivel de generalidad y una fuerza teórica que les permita ir más allá de los conflictos de su tiempo, pero, al mismo tiempo, estas teorías deben estar conectadas al mundo concreto y a sus problemas más complejos (Nussbaum, 2006), de ahí que como indica la misma autora un mínimo social básico es indispensable ya que este constituye lo que las personas son realmente son capaces de hacer o ejercer dentro de la sociedad.

\section{Referencias bibliográficas}

Barbarosch, E. (2013). Teorías de la Justicia y la Metaética Contemporánea. Buenos Aires: La Ley; Departamento de Publicaciones de la Facultad de Derecho y Ciencias Sociales de la Universidad de Buenos Aires.

Berger, P., \& Luckmann, T. (1993). La construcción social de la realidad. Buenos Aires: Amorrortu.

Cárcova, C. M. (2002). Justicia como equidad o sociedad como conflicto (Una lectura crítica del liberalismo político). Revista de Ciencias Sociales, 51-66. 
Cárcova, C. M. (NF). La norma fundamental es una ficción. Profesor. Titular Emérito - UBA.

CEPAL. (23 de octubre de 2021). https://www.cepal.org. Obtenido de Comisión Económica para América Latina y el Caribe: https://www.cepal.org/es/comunicados/pandemiaprovoca-aumento-niveles-pobreza-sin-precedentes-ultimas-decadas-impacta

Chul Han, B. (2017). La sociedad del cansancio. Argentina:Editorial Herder.

Colmenarejo Fernández, R. (2013). La idea de la justicia de Amartya Sen, un tratado sobre la injusticia. Revista de Fomento Social, 43-58. Obtenido de https://www.revistadefomentosocial.es/rfs/article/download/1725/324

Francisco Caballero, J. (2006). La Teoría de la Justicia de John Rawls. Voces y contextos, 1-22. Obtenido de https://ibero.mx/iberoforum/2/pdf/francisco_caballero.pdf

Gassner, M., \& Olechowski, T. (2014). Teoría Egológica del Derecho versus Teoría Pura del Derecho - Cossio Versus Kelsen. Revista Anales de la Facultad de Ciencias Jurídicas y Sociales. U.n.l.p., 296-306. Obtenido de http://sedici.unlp.edu.ar/bitstream/handle/10915/43652/Documento_completo.pdf? sequence $=1$

Gómez Domínguez, P., Candelaria Rivera, G., Pérez Latorre, O., \& Gómez Puertas, L. (2016). Las narrativas del ataque contra Charlie Hebdo. Un estudio de la cobertura periodística internacional. Estudios sobre el Mensaje Periodístico, 417 - 435. Obtenido de https://www.researchgate.net/publication/318350553_Las_narrativas_del_ataque_c ontra_Charlie_Hebdo_Un_estudio_de_la_cobertura_periodistica_internacional

Gramsci, A. (1978). Antología. Siglo XXI.

Marí, E. E. (1986). Racionalidad e imaginario social en el discurso del orden. Doxa.

Nozick, R. (1988). Anarquía, Estado y utopía. México: FCH.

Nussbaum, M. (2006). Las fronteras de la justicia: consideraciones sobre la exclusión. Barcelona: Ediciones Paidós Estado y Sociedad.

OIM. (2021). Informe de la OIM: las muertes en las rutas migratorias marítimas hacia Europa aumentaron más del doble en la primera mitad de 2021. Berlin: Organización Internacional para las Migraciones. Obtenido de https://www.iom.int/es/news/informe-de-la-oim-las-muertes-en-las-rutasmigratorias-maritimas-hacia-europa-aumentaron-mas-del-doble-en-la-primeramitad-de-2021-0

Oxfam. (24 de octubre de 2021). https://www.oxfam.org. Obtenido de https://www.oxfam.org/es/que-hacemos/temas/desigualdad-extrema-y-serviciossociales-basicos

Piscitelli, A. (1993). Ciencia en movimiento la construccion social de los hechos. Buenos Aires: Centro Editor de América Latina.

Rawls, J. (1971). Una teoría de la Justicia. Estados Unidos: Harvard University Press.

Ruiz, A. E. (2014). La letra y la ley Estudios sobre derecho y literatura. En Jorge E. Douglas Price, \& Carlos María Cárcova, Cuerpo/Cuerpos. Buenos Aires: Infojus.

Schuster, F. (2001). Exposición. Argentina: Homo Sapiens Ediciones.

Sen, A. (2011). La idea de la justicia. Buenos Aires: Taurus.

Žižek, S. (2003). El Espectro de la Ideología. En Ideología un Mapa de la Cuestión. México: Fondo de Cultura Económica. 